\title{
ON THE TYPE AND THE STANDARDFORM OF INDUCED UA REPRESENTATIONS
}

\author{
P.M. VAN DEN BROEK \\ Institute for Theoretical Physics, University of Nimegen, Nimegen, \\ The Netherlands
}

\begin{abstract}
We derive to which of the three types an irreducible UA representation which is obtained with the procedure of generalised induction belongs. We analyse the question whether or not the irreducible induced UA representations are on standardform. The results hold as well for induced PUA representations.
\end{abstract}

\section{INTRODUCTION}

It is well known from Wigner (1959) that the irreducible unitary-antiunitary (UA) representations of a group are naturally divided into three types and that they can be chosen from their equivalence classes in such a way that they have a special form, called standardform. It is very important if one deals with irreducible $U \Lambda$ representations that one knows to which type they belong and that they are on standardform; for instance the lemma of Schur and the orthogonality relations for matrix elements, generalised for irreducible UA representations, are different for each of the three types and can be formulated suitably only for irreducible $U A$ representations which are on standardform (see for instance [1])

A powerful method for the construction of a complete set of inequivalent irreducible UA representations of a group is the procedure of generalised induction, given by Shaw and Lever [2]. The aim of this paper is to derive to which of the types an irreducible UA representation which is obtained with this procedure belongs and to analyse the question under which conditions the irreducible induced UA representations are on standardform.

Although the most general form of representations occurring in plysical applications are projective (P)UA representations rather than UA representations we restrict ourselves here to UA representations in order to avoid tedious calculations with factor systems. It is, lowever, a straightforward exercise to show that all the results of this paper hold as well for induced PUA representations.

\section{PRELIMINARILS}

Let $G$ be a finite group and $G_{0}$ a subgroup of $G$ of index 2 . A UA representation of $G$ with

I.etters in Mathematical Physics 3 (1979) $151 \quad 159$. 
respect to $G_{0}$ is a mapping $D$ from $G$ into the unitary matrices of some dimension $n$ such that

$$
D(g) D^{g}\left(g^{\prime}\right)=D\left(g g^{\prime}\right), \quad \forall g, g^{\prime} \in G
$$

where $D^{g}$ is defined by

$$
D^{g}=\left\{\begin{array}{lll}
D & \text { if } & g \in G_{0} \\
D^{*} & \text { if } & g \notin G_{0}
\end{array} .\right.
$$

In the following we shall drop the plarase 'with respect to $G_{0}$ '.

Two UA representations $D_{1}$ and $D_{2}$ of $G$ are equivalent if there exists a unitary matrix $\mathrm{U}$ such that

$$
D_{1}(g) U^{g}=\mathrm{U} D_{2}(g) \quad \forall g \in G .
$$

A UA representation $D$ is reducible if it is equivalent with a UA representation $D^{\prime}$ which has the form

$$
D^{\prime}(g)=\left(\begin{array}{cc}
D_{1}(g) & 0 \\
0 & D_{2}(g)
\end{array}\right) \quad \forall g \in G
$$

According to Wigner [3] the irreducible UA representations are divided into three types: the restriction $D \downarrow G_{0}$ of an irreducible UA representation $D$ of $G$ to $G_{0}$, which is a unitary representation of $G_{0}$, is irreducible (Type $)$ ), is reducible into two equivalent irreducible components (Type II), or is reducible into two inequivalent irreducible components (Type III).

Let $a$ be a fixed element of $G \backslash G_{0}$. An irreducible UA representation of $G$ of Type II is equivalent with a $U A$ representation which has the form

$$
D(g)=\left(\begin{array}{cc}
\Delta(g) & 0 \\
0 & \Delta(g)
\end{array}\right) \quad \forall g \in G_{0} ; \quad D(a)=\left(\begin{array}{cc}
0 & U \\
-U & 0
\end{array}\right) .
$$

An irreducible UA representation of $G$ of Type III is equivalent with a UA representation which has the form

$$
D(g)=\left(\begin{array}{cc}
\Delta(g) & 0 \\
0 & \bar{\Delta}(g)
\end{array}\right) \quad \forall g \in G_{0} ; \quad D(a)=\left(\begin{array}{cc}
0 & \Delta\left(a^{2}\right) \\
\Pi & 0
\end{array}\right)
$$

where $\bar{\Delta}(g)$ is given by $\Delta(g)=\Delta^{*}\left(a^{-1} g a\right)$.

All irreducible LA representation is said to be on standardform if it is of Type $I$, if it is of Type II and satisfies eqn. (2.5) or if it is of Type III and satisfies eqn. (2.6). So each irreducible UA representation is equivalent with an irreducible UA representation which is on standardform. 
Now let $H$ be an invariant subgroup of $G$ which belongs to $G_{0}$, let $K$ be the quotient group $G / H$ and let $K_{0}$ be the quotient group $G_{0} / H$. The elements of $H$ will be denoted by $a, b, c, \ldots$ and elements of $K$ by $\alpha, \beta, \gamma, \ldots$ Take for each coset $\alpha \in K$ a representative $r(\alpha)$, and let $r(\epsilon)=e$, if $e$ and $e$ are the unit elements of $K$ and $G$, respectively. Then each element of $G$ can uniquely be written as $(a, \alpha)$ if we define

$$
(a, \alpha)=\operatorname{ar}(\alpha)
$$

We suppose that the irreducible unitary representations of $H$ are known. From these a complete set of inequivalent irreducible UA representations of $G$ is obtained with the procedure of generalised induction [2], which will be briefly reviewed here.

Let $\Delta$ be an irreducible unitary representation of $H$. For each $\alpha \in K$ define the irreducible unitary representation $\Delta_{\alpha}$ of $H$ by

$$
\Delta_{c}(a)=\Delta^{\alpha}\left(r^{-1}(\alpha) a r(\alpha)\right)
$$

where $\Delta^{\alpha}$ is a shorthand symbol for $\Delta^{(e, \alpha)}$.

The elements $\alpha \in K$ for which $\Delta$ and $\Delta_{\alpha}$ are equivalent form a subgroup $\bar{K}$ of $K$. The little group $L(G)$ of $\triangle$ is defined by

$$
L(G)=\{(a, \alpha) \mid a \in H, \alpha \in \bar{K}\}
$$

$L(G)$ is a subgroup of $G$ which contains $H$ but is not necessarily an invariant subgroup. The set $\left\{\triangle_{\alpha} l \alpha \in K\right\}$ is called the $G$-orbit of $\triangle$.

For any $\alpha \in \bar{K}$ there exists a unitary operator $\mathrm{L}(\alpha)$ such that

$$
\Delta(a)=\mathrm{U}(\alpha) \Delta_{\alpha}(a) \mathrm{U}^{-1}(\alpha) \quad \forall a \in H
$$

Define $V(a, \alpha)$ by

$$
V(a, \alpha)=\Delta(a) \mathrm{U}(\alpha)
$$

for each $(a, \alpha) \in L(G)$. Then it can be shown that

$$
V(\alpha, \alpha) V^{\alpha}(b, \beta)=\mu(\alpha, \beta) V((\alpha, \alpha)(b, \beta))
$$

for some mapping $\mu: \bar{K} \times \bar{K} \rightarrow \mathrm{U}(1)$, which means that $V$ is a PU(A) representation of $L(G)$ (i.e. a PUA representation if $L(G) \notin G_{0}$ and a PU representation if $L(G) \subseteq G_{0}$ ) whose factor system $\mu$ is a factor system of $K_{0}$.

Now let $E$ be an irreducible PU(A) representation of $\bar{K}$ with factor system $\mu^{*}$ :

$$
E(\alpha) E^{\alpha}(\beta)=\mu^{*}(\alpha, \beta) E(\alpha \beta)
$$


then, with the definition

$$
\mathscr{D}(a, \alpha)=V(a, \alpha) \otimes E(\alpha) \quad \forall a \in H, \forall \alpha \in \bar{K}
$$

$\mathscr{D}$ is an irreducible U(A) representation of $I .(C)$.

Let $g_{1}=e, g_{2}, \ldots ., g_{p}$ be left-coset-representations of $G$ with respect to $L(G)$. Then $G=$ $\Sigma_{i=1}^{p} g_{i} L(G)$.

For each U(A) rep $\mathscr{D}$ of $L(G)$ the induced UA rep $\mathscr{P} \uparrow G$ is defined by

$$
(\mathscr{D} \uparrow G)_{j l, k s}(g)=\mathscr{D}_{t s}^{g_{j}}\left(g_{j}^{-1} g g_{k}\right) \delta\left(g_{j}^{-1} g g_{k}, L(G)\right)
$$

where $\delta(g, L(G))$ is defined by

$$
\delta(g, L(G))=\left\{\begin{array}{lll}
1 & \text { if } & g \in L(G) \\
0 & \text { if } & g \notin L(G)
\end{array}\right.
$$

A complete set of inequivalent irreducible UA representations is obtained in the fullowing way:

(i) Take one irreducible unitary representation $\triangle$ of $H$ from each $G$-orbit.

(ii) For each $\Delta$, obtain the $U(A)$ representations of $L(G)$ given by eqn. (2.15) where $E$ runs through a complete set of inequivalent irreducible $\mathrm{PU(A)}$ representations of $\vec{K}$ with factor system $\mu^{*}$.

(iii) Induce these $\mathrm{U}(\mathrm{A})$ representations to $G$.

Note that the irreducible unitary representations of $G_{0}$ can be obtained from the irreducible unitary representations of $H$ in exactly the same way: one only has to replace $G$ by $G_{0}$ and $K$ by $K_{0}$ in the method described above. In fact, we wrote $L(G)$ and $G$-orbit instead of just $L$ and orbit in order to be able to distinguish between $L(G)$ and $L\left(G_{0}\right), G$-orbit and $G_{0}$-orbit.

\section{THE TYPE AND THE STANDARDIORM OF INDUCEDUA REPRESENTATIONS}

Let $\Delta$ be an irreducible unitary representation of $H, L(G)$ its little group, $\mathscr{P}$ the irreducible $U(A)$ representation of $L(G)$ given by eqn. (2.14) where $E$ is an irreducible PU(A) representation of $\bar{K}$ with factor system $\mu^{*}$, and $\mathscr{D} \uparrow G$ the irreducible UA representation of $G$ given by eqn. (2.15). The first question we will answer in this section is: what is the type of $\mathscr{D} \uparrow G$ ? The second question is: can we manage that $\mathscr{D} \uparrow G$ is on standardform?

To answer these questions we have to distinguish two different cases: $L(G)$ belongs to $G_{0}$ or $L(G)$ does not belong to $G_{0}$. Let us first consider the case that $L(G)$ belongs to $G_{0}$. Then $L(G)$ equals $L\left(G_{0}\right)$. The $G$-orbit of $\triangle$ contains two $G_{0}$-orbits of $\Delta$ : the $G_{0}$-orbit of $\Delta$ and the $G_{0}$-orbit of $\bar{\Delta}$. The restriction of $\mathscr{Q} \uparrow G$ to $H$ (notation: $(\mathscr{P} \uparrow G) \downarrow H$ ) contains as irreducible components only elements of the $G$-orbit of $\Delta$; each element of this orbit accurs an equal number of times. But since $\left((\mathscr{D} \uparrow G) \downarrow G_{0}\right) \downarrow H=(\mathscr{D} \uparrow G) \downarrow H$ the restriction of $(\mathscr{P} \uparrow G) \downarrow G_{0}$ to $H$ contains as ir reducible components the elements of two different $G_{0}$-orbits. This means that $(\mathscr{D} \uparrow G) \downarrow G_{0}$ must contain at least two inequivalent irre- 
ducible components; so $\mathscr{P} \uparrow G$ must be of Type III.

Let $g_{1}=e, g_{2}, \ldots . g_{p}$ be left-coset-representatives of $G_{0}$ with respect to $L(G)$. Then we may choose the left-coset-representatives of $G$ with respect to $L(G)$ to be $g_{1}=e, g_{2}, \ldots, g_{p}, a, a g_{2}, \ldots$, $a g_{p}$. Define

$$
g_{j m}(j=1, \ldots, p ; m=1,2) \text { by } g_{j 1}=g_{i} \text { and } g_{i 2}=a g_{j} \text {. }
$$

Then $G=\sum_{j=1}^{p} \Sigma_{m=1}^{2} g_{j m} L(G)$. Equation (2.15) can now be written as

$$
(\mathscr{P} \uparrow G)_{j m t, k n s}(g)=\mathscr{O}_{T s}^{g_{j m}}\left(g_{j m}^{-1} g g_{k n}\right) \delta\left(g_{i m}^{-} g g_{k m}, L(G)\right)
$$

For $g \in G_{0}$ the blocks $(\mathscr{D} \uparrow G)_{j 1 t, k 2 s}(g)$ and $(\mathscr{D} \uparrow G)_{j 2 t, k 1 s}(g)$ are equal to zero:

$$
(\mathscr{D} \uparrow G)_{j 1 t, k 1 s}(g)=\mathscr{D}_{t s}\left(g_{j 1}^{-1} g g_{k l}\right) \delta\left(g_{j 1}^{-1} g g_{k 1}, L\left(G_{0}\right)\right)=\left(\mathscr{D} \uparrow G_{0}\right)_{j t, k s}(g)
$$

and

$$
(\mathscr{P} \uparrow G)_{j 2 t, k 2 s}(g)=\mathscr{G}_{i s}^{*}\left(g_{j 2}^{-1} g g_{k 2}\right) \delta\left(g_{j 2}^{-1} g g_{k 2} . L\left(G_{0}\right)\right)=\left(\mathscr{D} \uparrow G_{0}\right)_{j, k s}^{*}\left(a^{-1} g a\right) .
$$

The blocks $(\mathscr{D} \uparrow G)_{j 1, k 1 s}(a)$ and $(\mathscr{Q} \uparrow G)_{j 2 r, k 2 s}(a)$ are equal to zero;

$$
(\mathscr{D} \uparrow G)_{j 1 t, k 2 s}(a)=\mathscr{D}_{t s}\left(g_{j 1}^{-1} a g_{k 2}\right) \delta\left(g_{j 1}^{-1} a g_{k 2}, L\left(G_{0}\right)\right)=\mathscr{D}_{t s}\left(g_{j 1}^{-1} a^{2} g_{k 1}\right) \delta\left(g_{j 1}^{-1} a^{2} g_{k 1}, L\left(G_{0}\right)\right)
$$

and

$$
(\mathscr{D} \uparrow G)_{j 2 t, k 1 s}(a)=\mathscr{D}_{i s}^{*}\left(g_{j 2}^{-\mathrm{I}} a g_{k 1}\right) \delta\left(g_{i 2}^{-1} a g_{k 1}, L\left(G_{0}\right)\right)=\mathscr{D}_{i s}^{*}\left(g_{l}^{-1} g_{k}\right) \delta\left(g_{j}^{-1} g_{k}, L\left(G_{0}\right)\right)=\delta_{j k} \delta_{t s}
$$

So with this choice of left-coset-representatives we obtained the following blockform for $\mathscr{D} \uparrow G$ :

$$
\begin{aligned}
& (\mathscr{D} \uparrow G)(g)=\left(\begin{array}{cc}
\left(\mathscr{Q} \uparrow G_{0}\right)(g) & 0 \\
0 & \left(\mathscr{D} \uparrow G_{0}\right) *\left(a^{-1} g a\right)
\end{array}\right) \quad \forall g \in G_{0}: \\
& (\mathscr{D} \uparrow G)(a)=\left(\begin{array}{cc}
0 & \left(\mathscr{P} \uparrow G_{0}\right)\left(a^{2}\right) \\
\rrbracket & 0
\end{array}\right)
\end{aligned}
$$

which is exactly the standardform for an irreducible UA representation of Type III. It is obvious that with an arbitrary choice of the left-coset-representatives $\mathscr{D} \uparrow G$ is not on standardform in general.

Now let us consider the case that $L(G)$ does not belong to $G_{0}$. Then $L\left(G_{0}\right)$ is equal to 
$L(G) \cap G_{0}$ and is a subgroup of $L(G)$ of index 2 . The $G$-orbit of $\Delta$ is equal to the $G$-orbit of $\Delta$. Let $\bar{K}_{0}$ be the quotient group $L\left(G_{0}\right) / H$. $\bar{K}_{0}$ is a subgroup of $\bar{K}$ of index 2 . Let $\alpha_{0}$ be an element of $\bar{K} \backslash \bar{K}_{0}$. The element $a \in G \backslash G_{0}$ we choose to be $a=\left(e, \alpha_{0}\right)$. The left-coset-representatives $g_{1}=e, g_{2}, \ldots, g_{p}$ of $G$ with respect to $L(G)$ are chosen to belong to $G_{0}$, which means that they are also left-coset-representatives of $G_{0}$ with respect to $L\left(G_{0}\right)$. This means that the relation

$$
(\mathscr{D} \uparrow G) \downarrow G_{0}=\left(\mathscr{D} \downarrow L\left(G_{0}\right)\right) \uparrow G_{0}
$$

is valid with this choice of left-coset-representatives. $\mathscr{D}$ is given by eqn. (2.14) where $E$ is an irreducible PUA representation of $\bar{K}$ with respect to $\bar{K}_{0}$ with factor system $\mu^{*}$.

The type and the standardform of an irreducible PUA representation is defined in the same way as the type and the standardform of an irreducible UA representation, the only difference being the appearance of the factor system in eqn. (2.6) which reads

$$
D(g)=\left(\begin{array}{cc}
\Delta(g) & 0 \\
0 & \bar{\Delta}(g)
\end{array}\right) \quad \forall g \in G_{0} ; \quad D(a)=\left(\begin{array}{cc}
0 & \sigma(a, a) \Delta\left(a^{2}\right) \\
\square & 0
\end{array}\right)
$$

where $\bar{\Delta}(g)$ is given by $\Delta(g)=\sigma^{*}\left(a, a^{-1} g a\right) \sigma(g, a) \triangle^{*}\left(a^{-1} g a\right)$ and $\sigma$ is the factor system of $D$.

$E \downarrow \widetilde{K}_{0}$ is irreducible, consists of two equivalent irreducible components or consists of two inequivalent irreducible components if $E$ is of Type I, II or III, respectively. From eqn. (2.14) it then follows that also $\mathscr{D} \downarrow L\left(G_{0}\right)$ is irreducible, consists of two equivalent irreducible components or consists of two inequivalent components respectively. But from eqn. (3.7) it then follows that the same holds for $(\mathscr{D} \uparrow G) \downarrow G_{0}$. This shows that $\mathscr{D} \uparrow G$ is of Type I, II or III if $E$ is of Type I, II or III, respectively

Suppose that $E$ is of Type II and is on standardform. Then we may write

$$
E(\alpha)=\left(\begin{array}{cc}
F(\alpha) & 0 \\
0 & F(\alpha)
\end{array}\right) \quad \forall \alpha \in K_{0} ; \quad E\left(\alpha_{0}\right)=\left(\begin{array}{cc}
0 & A \\
-A & 0
\end{array}\right)
$$

or, using double indices

$$
E(\alpha)_{m p, n q}=F(\alpha)_{m n} \delta_{p q} \quad \forall \alpha \in K_{0} ; E\left(\alpha_{0}\right)_{m p, n q}=A_{m n}\left(\delta_{1 p} \delta_{2 q}-\delta_{1 q} \delta_{2 p}\right)
$$

for $p, q=1,2 ; m, n=1, \ldots, \frac{1}{2} \operatorname{dim} E$ and where $F$ is an irreducible PU representation of $\bar{K}_{0}$ with factor system $\mu^{*}$.

Equation (2.14) can be written as

$$
\mathscr{D}(a, \alpha)_{k m p, \ell n q}=V(a, \alpha)_{k \ell} E(\alpha)_{m p, n q} \quad \forall a \in H, \quad \forall \alpha \in \bar{K}
$$

Without loss of generality we may assume that the left-coset-representatives $g_{1}=e_{,} g_{2}, \ldots, g_{p}$ are given by $\left(e, \alpha_{1}\right),\left(e, \alpha_{2}\right), \ldots,\left(e, \alpha_{p}\right)$ where $\alpha_{1}=\epsilon, \alpha_{2}, \ldots, \alpha_{p}$ are left-coset-representatives of $K_{0}$ with respect to $\bar{K}_{0}$. Then eqn. $(2.15)$ can be written as:

$$
(\mathscr{D} \uparrow G)_{i k m p, j \ell n q}(a, \alpha)=\mathscr{A}_{k m p, \ell n q}\left(\left(e, \alpha_{1}\right)^{-1}(a, \alpha)\left(e, \alpha_{j}\right)\right) \delta\left(\alpha_{i}^{-1} \alpha \alpha_{j}, \bar{K}\right)
$$


For $\alpha \in \bar{K}_{0}$ this becomes, with eqns. (3.10) and (3.11)

$(\mathscr{D} \uparrow G)_{i k m p, j \ell n q}(a, \alpha)=V_{k \ell}\left(\left(e, \alpha_{1}\right)^{-1}(a, \alpha)\left(e, \alpha_{i}\right)\right) F\left(\alpha_{i}^{-1} \alpha \alpha_{j}\right)_{m+n} \delta_{p q} \delta\left(\alpha_{i}^{-1} \alpha \alpha_{j}, \bar{K}_{0}\right)$.

So if we write $\mathscr{O} \uparrow G$ in blockform with respect to the indices $p$ and $q$ we obtain

$$
(\mathscr{D} \uparrow G)(a, \alpha)=\left(\begin{array}{cc}
\left(\mathscr{D}^{\prime} \uparrow G_{0}\right)(a, \alpha) & 0 \\
0 & \left(\mathscr{Q}^{\prime} \uparrow G_{0}\right)(a, \alpha)
\end{array}\right) \quad \forall a \in H, \quad \forall \alpha \in \bar{K}_{0},
$$

where $\mathscr{D}^{\prime}$ is the irreducible unitary representation of $L\left(G_{0}\right)$ given by

$$
\mathscr{Q}^{\prime}(a, \alpha)=\left(V \downarrow L\left(G_{0}\right)\right)(a, \alpha) \otimes F(\alpha) \quad \forall a \in H, \quad \forall \alpha \in \bar{K}_{0}
$$

From the eqns. (3.11) and (3.12) it follows

$$
(\mathscr{D} \uparrow G)_{i k m p, j \ell q q}\left(e, \alpha_{0}\right)=V_{k \ell}\left(\left(e, \alpha_{i}\right)^{-1}\left(e, \alpha_{0}\right)\left(e, \alpha_{j}\right)\right) E_{m p, n q}\left(\alpha_{i}^{-1} \alpha_{0} \alpha_{j}\right) \delta\left(\alpha_{i}^{-1} \alpha \alpha_{k}, \bar{K}\right) .
$$

Since

$$
E\left(\alpha_{i}^{-1} \alpha_{0} \alpha_{j}\right)=\mu\left(\alpha_{i}^{-1} \alpha_{0} \alpha_{j} \alpha_{0}^{-1}, \alpha_{0}\right)\left(\begin{array}{cc}
0 & F\left(\alpha_{i}^{-1} \alpha_{0} \alpha_{j} \alpha_{0}^{-1}\right) A \\
F\left(\alpha_{i}^{-1} \alpha_{0} \alpha_{j} \alpha_{0}^{-1}\right) A & 0
\end{array}\right)
$$

it follows from eqn. (3.16) that the two diagonal blocks of $(\mathscr{D} \uparrow G)\left(e, \alpha_{0}\right)$ are equal to zero and that the two off-diagonal blocks only differ from each other by a minus sign. This shows that $\mathscr{D} \uparrow G$ is on standardform if we write the matrices in blockform with respect to the indices $p$ and $q$. This result is independent of the choice of the left-coset-representatives.

Now suppose that $E$ is of Type III and is on standardform. Then we may write

$$
E(\alpha)=\left(\begin{array}{cc}
F(\alpha) & 0 \\
0 & \bar{F}(\alpha)
\end{array}\right) \quad \forall \alpha \in \bar{K}_{0}
$$

where $\bar{F}(\alpha)$ is given by

$$
\bar{F}(\alpha)=\mu\left(\alpha_{0}, \alpha_{0}^{-1} \alpha \alpha_{0}\right) \mu^{*}\left(\alpha, \alpha_{0}\right) F^{*}\left(\alpha_{0}^{-1} \alpha \alpha_{0}\right) .
$$

Also for this case eqns. (3.11) and (3.12) are valid.

If we write $\mathscr{D} \uparrow G$ in bluckform with respect to the indices $p$ and $q$ we obtain

$$
(\mathscr{D} \uparrow G)(a, \alpha)=\left(\begin{array}{cc}
\left(\mathscr{D}^{\prime} \uparrow G_{0}\right)(a, \alpha) & 0 \\
0 & \left(\overline{\mathscr{D}}^{\prime} \uparrow G_{0}\right)(a, \alpha)
\end{array}\right),
$$

where $\mathscr{P}^{\prime}$ is given by eqn. $(3.15)$ and $\mathscr{W}^{\prime}$ is given by 


$$
\overline{\mathscr{D}}^{\prime}(a, \alpha)=\left(V \downarrow L\left(G_{0}\right)\right)(a, \alpha) \otimes \bar{F}(\alpha) \quad \forall a \in H, \quad \forall \alpha \in \bar{K}_{0} .
$$

Since in general $\left(\mathscr{D}^{\prime} \uparrow G_{0}\right)(a, \alpha)$ is not equal to $\left(\mathscr{D}^{\prime} \uparrow G_{0}\right)^{*}\left(\left(e, \alpha_{0}\right)^{-1}\left(e, \alpha_{0}\right)\right)$ we find that $\mathscr{D} \uparrow G$ is not on standardform in general. This difficulty may be overcome by finding the unitary transformation which brings $\mathscr{D} \uparrow G$ on standardform. However, if we realize that $\mathscr{D} \uparrow G$ is of Type IIJ and that the standardform of an irreducible UA representation (eqn. (2.6)) is known if one of the irreducible components of its restriction to the unitary subgroup is known we may forget about the induced representation $\mathscr{D} \uparrow G$ and consider instead the irreducible UA representation $D$ of $G$ given by

$$
D(a, \alpha)=\left(\begin{array}{cc}
\left(\mathscr{D}^{\prime} \uparrow G_{0}\right)(a, \alpha) & 0 \\
0 & \left(\mathscr{D}^{\prime} \uparrow G_{0}\right)^{*}\left(\left(e, \alpha_{0}\right)^{-1}(a, \alpha)\left(e, \alpha_{0}\right)\right)
\end{array}\right) \quad \forall a \in H, \quad \forall \alpha \in K_{0}
$$

and

$$
D\left(e, \alpha_{0}\right)=\left(\begin{array}{cc}
0 & \left(\mathscr{D}^{\prime} \uparrow G_{0}\right)\left(\left(e, \alpha_{0}\right)^{2}\right) \\
0 & 0
\end{array}\right)
$$

which is on standardform.

However, we must keep in mind that $D$ and $\mathscr{O} \uparrow G$ are equivalent but not equal in general.

\section{SUMMARY OI' THE RESULTS}

We distinguished two cases: $L(G)$ belongs to $G_{0}$ and $L(G)$ does not belong to $G_{0}$. If $L(G)$ belongs to $G_{0}$ then $\mathscr{D} \uparrow G$ is of Type III and, with a proper choice of left-coset-representatives and a proper order of the indices, is on standardform. If $L(G)$ does not belong to $G_{0}$ the type of $\mathscr{Q} \uparrow G$ is the same as the type of the irreducible PUA representation $E$ of $K$ which is used to obtain $\mathscr{P}$ (with eqn. (2.14)). If $\mathscr{D} \uparrow G$ and $E$ are of Type II then $\mathscr{P} \uparrow G$ is on standardform (with a proper order of the indices). If $\mathscr{D} \uparrow G$ and $E$ are of Type 111 then $\mathscr{D} \uparrow G$ is not on standardform in general, but in this case an equivalent $U A$ representation which is on standardform can be written down at once (eqn. (3.22)).

\section{ACKNOWLEDGEMENT}

I thark Dr T. Janssen for reading the manuscript.

\section{RLIERENCES}

1. Van den Broek, P.M., 'Clebsch-Gordan coefficients of finite magnetic groups', submitted for 
publication to J. Phys. A: Math. Gen.

2. Shaw, R, and Lever, J., Commun. Math. Phys. 38, 257-77 (1974).

3. Wigner, E.P., Group Theory and its Application to the Quantum Mechanics of Atomic Spectra, Academic Press, New York, 1959.

(Received December 18, 1978) 